\title{
A Remark on Eigenfunction Estimates by Heat Flow
}

\author{
Huabin Ge1, Yipeng Shi ${ }^{2}$ \\ ${ }^{1}$ Department of Mathematics, Beijing Jiaotong University, Beijing, China \\ ${ }^{2}$ College of Engineering, Peking University, Beijing, China \\ Email: hbge@bjtu.edu.cn,ypshi@coe.pku.edu.cn
}

Received 1 June 2016; accepted 24 June 2016; published 27 June 2016

Copyright (C) 2016 by authors and Scientific Research Publishing Inc.

This work is licensed under the Creative Commons Attribution International License (CC BY).

http://creativecommons.org/licenses/by/4.0/

(c) (i) Open Access

\begin{abstract}
In this paper, we consider $L^{\infty}$ estimates of eigenfunction, or more generally, the $L^{\infty}$ estimates of equation $-\Delta u=f u$. We use heat flow to give a new proof of the $L^{\infty}$ estimates for such type equations.
\end{abstract}

\section{Keywords}

\section{$L^{\infty}$ Estimates, Eigenfunction, Heat Flow}

\section{Introduction}

Let $\Omega \subset \mathbb{R}^{n}(n>2)$ be a bounded domain. Assume $u \in C^{2}(\Omega)$, we consider the Laplacian equation

$$
-\Delta u=f u \text {, }
$$

where $|f| \in L^{\infty}(\Omega)$ and $\Delta=\frac{\partial^{2}}{\partial x_{1}^{2}}+\cdots+\frac{\partial^{2}}{\partial x_{n}^{2}}$ with $x=\left(x_{1}, \cdots, x_{n}\right) \in \mathbb{R}^{n}$. This is a second order differential equation. If $f=\lambda$ is a constant, then $u$ is an eigenfunction with eigenvalue $\lambda$. By a standard Moser's iteration in [1]-[5], we have $L^{\infty}$ interior estimates of $u$ controlled by the $L^{p}$ norm of $u$ for $p>0$. In this paper, we use heat flow to consider the $L^{\infty}$ estimate and give a new proof of the $L^{\infty}$ estimates without using iteration. First, we recall the definition of the heat kernel. For any $x, y \in \mathbb{R}^{n}$ and $t>0$, let

$$
\rho_{t}(x, y)=\frac{1}{(4 \pi t)^{n / 2}} \mathrm{e}^{-\frac{|x-y|^{2}}{4 t}}
$$


be the heat kernel in $\mathbb{R}^{n}$. For fixed $y \in \mathbb{R}^{n}$, we know that

$$
\left(\partial_{t}-\Delta_{x}\right) \rho_{t}(x, y)=0
$$

where $\Delta_{x}$ is the standard Laplacian in $\mathbb{R}^{n}$ with respect to $x$. Our main result is the following

Theorem 1. Let $\Omega \subset \mathbb{R}^{n}$ be a bounded domain with $n>2$. Assume $u \in C^{2}(\Omega)$ and

$$
-\Delta u=f u
$$

on $\Omega$ with $|f| \leq A$. Then for any $p>n / 2$ and any compact sub-domain $\Omega^{\prime} \subset \Omega$, we have the interior $L^{\infty}$ estimate

$$
\sup _{x \in \Omega^{\prime}}|u| \leq C\left(p, n, A, \operatorname{dist}\left(\Omega^{\prime}, \partial \Omega\right)\right)\left(\int_{\Omega}|u|^{p}(y) \mathrm{d} y\right)^{1 / p},
$$

where $\operatorname{dist}\left(\Omega^{\prime}, \partial \Omega\right)$ is the distance of $\Omega^{\prime}$ and the boundary of $\Omega$.

Remark 2. Following from the proof, one can consider equation $-\Delta u=f u+g$ or $\sum_{i, j=1}^{n} a_{i j} \partial_{i} \partial_{j} u=f u$ by choosing appropriate kernel function $\rho_{t}$.

\section{Proving the Theorem}

To estimates on $\Omega^{\prime} \subset \Omega$, by the translation invariant and scaling invariant of the estimates, we only need to consider $\Omega=B_{1}(0)$ and $\Omega^{\prime}=B_{1 / 2}(0)$. By using heat flow, we have the following lemma.

Lemma 1. Let $B_{1}(0) \subset \mathbb{R}^{n}$ be a unite ball. Assume $u \in C^{2}\left(B_{1}(0)\right)$ and

$$
-\Delta u=f u
$$

on $B_{1}(0)$ with $|f| \leq A$. Then for any $y \in B_{1 / 2}(0)$, we have the interior $L^{\infty}$ estimate

$$
|u|(y) \leq C(n, A) \int_{B_{1}(0)} \frac{|u|(x)}{|x-y|^{n-2}} d y .
$$

Proof. Let $\phi(x)$ be a standard smooth cutoff function with support in $B_{1}(0)$ and $\phi \equiv 1$ on $B_{3 / 4}(0)$, moreover, $|\Delta \phi|+|\nabla \phi| \leq C(n)$. For any $y \in B_{1 / 2}(0)$, let

$$
\Psi_{t}(y)=\int_{B_{1}(0)} \phi(x) u(x) \rho_{t}(x, y) \mathrm{d} x .
$$

By the heat equation $\left(\partial_{t}-\Delta_{x}\right) \rho_{t}(x, y)=0$, integrating by parts, we have

$$
\begin{gathered}
\partial_{t} \Psi_{t}(y)=\int_{B_{1}(0)} \phi(x) u(x) \partial_{t} \rho_{t}(x, y) \mathrm{d} x \\
=\int_{B_{1}(0)} \phi(x) u(x) \Delta_{x} \rho_{t}(x, y) \mathrm{d} x \\
=\int_{B_{1}(0)} \Delta(\phi u) \rho_{t}(x, y) \mathrm{d} x \\
=\int_{B_{1}(0)}(\Delta \phi u+\phi \Delta u+2\langle\nabla \phi, \nabla u\rangle) \rho_{t}(x, y) \mathrm{d} x \\
=\int_{B_{1}(0)}\left(-\Delta \phi u+\phi \Delta u-2\left\langle\nabla \phi, \nabla \log \rho_{t}(x, y)\right\rangle u\right) \rho_{t}(x, y) \mathrm{d} x \\
=\int_{B_{1}(0)}\left(-\Delta \phi u+\phi f u+\frac{2}{t}\langle\nabla \phi, x-y\rangle u\right) \rho_{t}(x, y) \mathrm{d} x,
\end{gathered}
$$

where we use integrating by parts for term $2\langle\nabla \phi, \nabla u\rangle \rho_{t}(x, y)$ to get (7) from (6). By direct estimate, since $\nabla \phi(x)=0$ for $x \in B_{3 / 4}(0)$ and $y \in B_{1 / 2}(0)$, then $|\langle\nabla \phi, x-y\rangle| \leq C(n)$. Therefore, for $t \leq 1$, we have

$$
\left(|\Delta \phi|+t^{-1}|\langle\nabla \phi, x-y\rangle|\right) \rho_{t}(x, y) \leq C(n) t^{-1-n / 2} \mathrm{e}^{-C(n) / t} \leq C(n) .
$$


Hence, for $t \leq 1$ and noting that $|\phi| \leq 1$, we have

$$
\left|\partial_{t} \Psi_{t}(y)\right| \leq C(n) \int_{B_{1}(0)}|u|(x)+C(n) \int_{B_{1}(0)}|f|(x) \cdot|u|(x) \rho_{t}(x, y) \mathrm{d} x .
$$

Since $|f| \leq A$, then we have

$$
\left|\partial_{t} \Psi_{t}(y)\right| \leq C(n) \int_{B_{1}(0)}|u|(x)+C(n, A) \int_{B_{1}(0)}|u|(x) \rho_{t}(x, y) \mathrm{d} x .
$$

By the property of heat kernel, we have $\Psi_{0}(u)=u(y)$. Then we have

$$
\left|u(y)-\Psi_{1}(y)\right| \leq \int_{0}^{1}\left|\partial_{t} \Psi_{t}(y)\right| \mathrm{d} t \leq C(n) \int_{B_{1}(0)}|u|(x)+C(n, A) \int_{0}^{1} \int_{B_{1}(0)}|u|(x) \rho_{t}(x, y) \mathrm{d} x \mathrm{~d} t .
$$

On the other hand, as $n>2$, we have

$$
\int_{0}^{1} \rho_{t}(x, y) \mathrm{d} t=\int_{0}^{1}(4 \pi t)^{-n / 2} \mathrm{e}^{-|x-y|^{2} / 4 t} \mathrm{~d} t=(4 \pi)^{-n / 2} \int_{1}^{\infty} s^{-2+\frac{n}{2}} \mathrm{e}^{-s \frac{|x-y|^{2}}{4}} \mathrm{~d} s \leq C(n)|x-y|^{2-n} .
$$

Combining with $\left|\Psi_{1}(y)\right| \leq C(n) \int_{B_{1}(0)}|u|(x) \mathrm{d} x$, we have

$$
|u|(y) \leq C(n, A) \int_{B_{1}(0)} \frac{|u|(x)}{|x-y|^{n-2}} \mathrm{~d} x .
$$

Hence we finish the proof.

The following lemma is fundamental.

Lemma 2. For any $y \in B_{1}(0)$ and any $0<p<n$, we have

$$
\int_{B_{1}(0)} \frac{1}{|x-y|^{p}} \mathrm{~d} x \leq C(n, p) .
$$

Proof. Let $r_{i}=2^{-i}$ and $A_{i}=B_{r_{i-1}}(y) \backslash B_{r_{i}}(y)$. Then

$$
\begin{gathered}
\int_{B_{1}(0)} \frac{1}{|x-y|^{p}} \mathrm{~d} x \leq \sum_{i=0}^{\infty} \int_{A_{i}} \frac{1}{|x-y|^{p}} \mathrm{~d} x \leq \sum_{i=0}^{\infty} r_{i}^{-p} \int_{A_{i}} \mathrm{~d} x \\
\leq \sum_{i=0}^{\infty} r_{i}^{-p} C(n) r_{i-1}^{n} \leq C(n) \sum_{i=0}^{\infty} r_{i}^{n-p} \leq C(n, p) .
\end{gathered}
$$

Now we are ready to prove Theorem 1.

Proof of Theorem 1. Refmaintheorem. For any compact subset $\Omega^{\prime} \subset \Omega$, let $2 r:=\operatorname{dist}\left(\Omega^{\prime}, \partial \Omega\right)$. For any $x \in \Omega^{\prime}$, we have $B_{r}(x) \subset \Omega$. Consider equation

$$
-\Delta u=f u,
$$

on $B_{r}(x)$. By Lemma 1 , since the estimates are scaling invariant, we have

$$
|u(x)| \leq C(r, n, A) \int_{B_{r}(x)} \frac{|u|(y)}{|x-y|^{n-2}} \mathrm{~d} y \leq C(r, n, A)\left(\int_{B_{r}(x)}|u|^{p}(y) \mathrm{d} y\right)^{1 / p}\left(\int_{B_{r}(x)}|x-y|^{-\frac{p(n-2)}{p-1}}\right)^{(p-1) / p} .
$$

If $p>n / 2$, then $p(n-2) /(p-1)<n$. By Lemma 2 , we have

$$
|u|(x) \leq C(r, n, A, p)\left(\int_{B_{r}(x)}|u|^{p}(y) \mathrm{d} y\right)^{1 / p} \leq C(r, n, A, p)\left(\int_{\Omega}|u|^{p}(y) \mathrm{d} y\right)^{1 / p} .
$$

Hence we finish the proof.

\section{Acknowledgements}

The research is supported by National Natural Science Foundation of China under grant No.11501027. The first author would like to thank Dr. Wenshuai Jiang, Xu Xu for many helpful conversations. 


\section{References}

[1] Evans, L.C. (1998) Partial Differential Equations, Graduate Studies in Mathematics, 19. American Mathematical Society, Providence.

[2] Gilbarg, D. and Trudinger, N.S. (2001) Elliptic Partial Differential Equations of Second Order. Reprint of the 1998 Edition, Springer-Verlag, Berlin.

[3] Han, Q. and Lin, F. (2011) Elliptic Partial Differential Equations. 2rd Edition, Courant Lecture Notes in Mathematics, 1. Courant Institute of Mathematical Sciences, American Mathematical Society, New York, Providence.

[4] Moser, J. (1964) A Harnack Inequality for Parabolic Differential Equations. Communications on Pure and Applied Mathematics, 17, 101-134. http://dx.doi.org/10.1002/cpa.3160170106

[5] Moser, J. (1961) On Harnack’s Theorem for Elliptic Differential Equations. Communications on Pure and Applied Mathematics, 14, 577-591. http://dx.doi.org/10.1002/cpa.3160140329

\section{Submit or recommend next manuscript to SCIRP and we will provide best service for you:}

Accepting pre-submission inquiries through Email, Facebook, Linkedin, Twitter, etc A wide selection of journals (inclusive of 9 subjects, more than 200 journals)

Providing a 24-hour high-quality service

User-friendly online submission system

Fair and swift peer-review system

Efficient typesetting and proofreading procedure

Display of the result of downloads and visits, as well as the number of cited articles

Maximum dissemination of your research work

Submit your manuscript at: http://papersubmission.scirp.org/ 